\title{
CONJUGACY SEPARATING REPRESENTATIONS OF FREE GROUPS
}

\author{
B. A. F. WEHRFRITZ
}

\begin{abstract}
If $G$ is a free group and $g$ is an element of $G$ we show that there exists a residually finite (commutative) integral domain $R$ and a faithful matrix representation $\rho$ of $G$ over $R$ of finite degree such that the conjugacy class of $g \rho$ in $G \rho$ is closed in the topology induced on $G \rho$ by the Zariski topology on the full matrix algebra. It follows that free groups are conjugacy separable, a result obtained by a number of authors, see [1], [5] and [6].
\end{abstract}

If $G$ is a free group and $g$ is an element of $G$ then our main result states that there exists a residually finite (commutative) integral domain $R$, an integer $n$ and a faithful representation $\rho$ of $G$ into $G L(n, R)$ such that the set $g^{G} \rho$ of conjugates of $g \rho$ in $G \rho$ is closed in $G \rho$ in the topology induced on $G \rho$ by the Zariski topology on the full matrix algebra $R_{n}$. (For a description of the Zariski topology see Chapter 5 of [8].)

It is a simple lemma that any group with this property is conjugacy separable, where the latter concept is defined as follows. An element $g$ of a group $G$ is conjugacy distinguished in $G$ if for each element $x$ of $G$ that is not conjugate to $g$ there exists a homomorphism $\phi$ of $G$ into a finite group with $x \phi$ and $g \phi$ not conjugate in $G \phi$. A group is conjugacy separable if each of its elements is conjugacy distinguished. Thus we obtain in particular yet another proof (see [1], [5] and [6] for others) of the conjugacy separability of free groups. Not that this leads to a better proof, but our result is stronger; that not every conjugacy separable group satisfies the conclusion of the above theorem is a triviality.

Let $G$ be any group. $G$ can be made into a topological group by specifying the subgroups of $G$ of finite index to be a base of the open neighbourhoods of the identity. We call this topology the profinite topology on $G$. It is well known, and very simple to show, that an element $g$ of $G$ is conjugacy distinguished in $G$ if and only if $g^{G}$ is closed in the profinite topology. A Zariski topology on $G$ induced by a faithful representation tends to be coarser than the profinite topology.

LEMMA 1. Let $R$ be an integral domain and $\left\{a_{i}: i \in I\right\}$ a set of ideals of $R$

Received by the editors October 10, 1972.

AMS (MOS) subject classifications (1970). Primary 20E05, $20 \mathrm{H} 20$.

Key words and phrases. Free groups, conjugacy separable, faithful representations.

(c) American Mathematical Society 1973 
intersecting in $\{0\}$. If $S$ is a subset of $R_{n}$ with Zariski closure $\bar{S}$ in $R_{n}$, then

$$
S \subseteq \bigcap_{i \in I}\left(S+\left(\mathfrak{a}_{i}\right)_{n}\right) \subseteq S .
$$

Proof. Trivially $S \subseteq \bigcap\left(S+\left(\mathfrak{a}_{i}\right)_{n}\right)$. Let $f$ be an element of the polynomial ring $R\left[X_{i j}: i, j=1,2, \cdots, n\right]$ annihilating $S$ (under the obvious action of substitution). If $x \in \bigcap\left(S+\left(a_{i}\right)_{n}\right)$ and $i \in I$ then $x=s+k$ for some $s \in S$ and $k \in\left(a_{i}\right)_{n}$. Then modulo $a_{i}$ we have $f(x) \equiv f(s) \equiv 0$ and so $f(x) \in \bigcap a_{i}=\{0\}$. Therefore $x \in S$.

LEMMA 2. Let $R$ be a residually finite integral domain and $G$ a subgroup of $G L(n, R)$. Then every Zariski closed subset of $G$ is profinitely closed in $G$. In particular if for some $x \in G$ the conjugacy class $x^{G}$ is Zariski closed in $G$ then $x$ is conjugacy distinguished in $G$.

Proof. There exists a set $\left\{a_{i}: i \in I\right\}$ of ideals of $R$ such that $\bigcap a_{i}=\{0\}$ and such that each $R / \mathfrak{a}_{i}$ is finite. If $S$ is Zariski closed in $G$ then by Lemma 1 $S=G \cap \bigcap_{i}\left(S+\left(\mathfrak{a}_{i}\right)_{n}\right)=\bigcap_{i} S K_{i}$ where $K_{i}=G \cap\left(1+\left(\mathfrak{a}_{i}\right)_{n}\right)$. Since $K_{i}$ is a normal subgroup of $G$ of finite index the lemma is proved.

Lemma 3. Let $G$ be a group, $H$ a free normal subgroup of $G$ of finite index dividing $m$ and $g$ an element of $G$ of infinite order such that $G=\langle g, H\rangle$. If $\mu$ is the map of $G$ into itself given by $x \mu=x^{m}$, then $g^{G}=\left(g^{m}\right)^{G} \mu^{-1} \cap g H$.

Proof. Since $G / H$ is cyclic the right-hand side certainly contains $g^{a}$. Let $k \in\left(g^{m}\right)^{G} \mu^{-1} \cap g H$. Then $k=g h$ for some $h \in H$. Now $g H$ is a normal subset of $G$, so replacing $k$ by a conjugate if necessary we may assume that $k^{m}=g^{m}$. Since $g$ and $g h$ both centralize $g^{m}$, so $h$ does too. Now the freeness of $H$ implies that $C_{H}\left(g^{m}\right)$ is cyclic, so for some $c \in H$ and some integers $r$ and $s$ we have $g^{m}=c^{r}$ and $h=c^{s}$. Trivially $g$ centralizes $c^{r}$, so $c^{r}=\left(c^{g}\right)^{r}$. But $H$, being free, is a group with unique root extraction, so $c=c^{g}$. Thus $h=h^{g}$ and so $h^{m}=(g h)^{m} g^{-m}=1$. This implies that $h=1$ and hence $k=g \in g^{a}$.

THEOREM 1. Let $G$ be a free group and $g$ an element of $G$. Then there exists a ring $R$ of the form $R=Z\left[X, Y^{-1}\right]$, where $X$ is a set of indeterminates with $|X| \leqq|G|$ and $Y$ is a subset of $X$ and a faithful matrix representation $\rho$ of $G$ over $R$ of finite degree such that $g^{G} \rho$ is Zariski closed in $G \rho$.

Proof. By a theorem of M. Hall [2, Theorem 5.1 and proof] there exist subgroups $H$ and $K$ of $G$ with $(G: H)=m$ finite and $H=\langle g\rangle * K$. Choose a basis $\left\{g_{\alpha}: \alpha \in \Lambda\right\}$ of the free group $K$.

Let $c$ denote zero or a prime. Set $P_{0}=Z, P_{c}=G F(c)$ for $c>0$, and let

$$
R_{c}=P_{c}\left[x_{\alpha}(i, j), \operatorname{det} x_{\alpha}, x_{\alpha}(1,1)^{-1},\left(\operatorname{det} x_{\alpha}\right)^{-1}: 2 \leqq i+j \leqq 3, \alpha \in \Lambda\right]
$$

where $\left\{x_{\alpha}(i, j), \operatorname{det} x_{\alpha}: 2 \leqq i+j \leqq 3, \alpha \in \Lambda\right\}$ is a family of independent 
indeterminates over $P_{c}$,

$$
x_{\alpha}(2,2)=x_{x}(1,1)^{-1}\left(\operatorname{det} x_{\alpha}+x_{\alpha}(1,2) x_{\alpha}(2,1)\right)
$$

and $x_{\alpha}$ denotes the 2 by 2 matrix of the $x_{\alpha}(i, j)$. The homomorphism $\tau_{c}$ of $H$ into $G L\left(2, R_{c}\right)$ determined by $g \tau_{c}=\left(\begin{array}{ll}1 & 0 \\ 1 & 1\end{array}\right)$ and $g_{\alpha} \tau_{c}=x_{\alpha}$ for $\alpha \in \Lambda$ is one-to-one on $K$ for every $c$ and is one-to-one on $H$ for $c=0$. Further for every $c$ we have that $H \tau_{c}$ is the free product of $\left\langle g \tau_{c}\right\rangle$ and $K \tau_{c}$. These facts about $\tau_{c}$ follow easily from results of Nisnevič [4] or from [7, §3]. Write $H_{c}$ for $H \tau_{c}, K_{c}$ for $K \tau_{c}$ and $g_{c}$ for $g \tau_{c}$.

We claim that $\left\langle g_{c}\right\rangle^{H_{c}}=\left\{h^{-1} g_{c}^{i} h: h \in H_{c}, i \in Z\right\}$ is exactly the set of unipotent elements of $H_{c}$, a result that will clearly imply that $\left\langle g_{c}\right\rangle^{H_{c}}$ is Zariski closed in $H_{c}$. Since by definition $g_{c}$ is unipotent, every element of $\left\langle g_{c}\right\rangle^{H_{c}}$ is unipotent. Let $u$ be any unipotent element of $H_{c}$ and consider first the case $c>0$. Then $u$ has finite order and so does $g_{c}$. Thus the decomposition $H_{c}=\left\langle g_{c}\right\rangle * K_{c}$ and 4.1 .4 of [3] imply that some conjugate of $u$ lies in $\left\langle g_{c}\right\rangle$.

We now consider $H_{0}$. Choose a prime $c$ greater than the length of the normal form of $u$ relative to the decomposition $H_{0}=\left\langle g_{0}\right\rangle * K_{0}$. The natural projection of $R_{0}$ onto $R_{c}$ (reduction modulo $c$ ) induces a homomorphism $\pi$ of $H_{0}$ onto $H_{c}$ satisfying $\tau_{0} \pi=\tau_{c}$. Since $\pi$ is induced by a ring homomorphism $u \pi$ is also unipotent and hence by the previous paragraph lies in $\left\langle g_{c}\right\rangle^{H_{c}}$. Thus $u^{x} \pi=g_{c}^{i}$ for some $x \in H_{0}$ and some $i$ with $0 \leqq i<c$. A cyclically reduced normal form of $u$ has length less than $c$ and its image under $\pi$ reduces cyclically to $g_{c}^{i}$, see [3, Theorem 4.2]. It follows that $g_{0}^{i}$ is a cyclically reduced normal form of $u$ and so $u \in\left\langle g_{0}\right\rangle^{H_{0}}$. We have now shown that $\left\langle g_{0}\right\rangle^{H_{0}}$ is Zariski closed in $H_{0}$.

Let $R=R_{0}\left[\xi, \xi^{-1}\right]$ where $\xi$ is an indeterminate over $R_{0}$. Define a homomorphism $\tau$ of $H$ into $G L(3, R)$ by $g \tau=\operatorname{diag}\left(g_{0}, \xi\right)$ and $g_{\alpha} \tau=$ $\operatorname{diag}\left(x_{\alpha}, 1\right)$ for $\alpha \in \Lambda$. Trivially $\tau$ is a faithful representation of $H$ such that $\langle g\rangle^{H} \tau$ is Zariski closed in $H \tau$. Also $g^{H} \tau$ consists of precisely those elements of $\langle g\rangle^{H} \tau$ whose $(3,3)$ th entry is $\xi$. Therefore $g^{H} \tau$ is Zariski closed in $H \tau$.

Choose a right transversal $1=x_{1}, x_{2}, \cdots, x_{m}$ of $H$ to $G$. For each $x$ in $G$ there exists an element $\sigma$ of $\operatorname{Sym}(m)$ and elements $a_{i}$ of $H$ such that $x_{i} x=$ $a_{i} x_{i \sigma}$, for $i=1,2, \cdots, m$. Define a map $\rho$ of $G$ into $G L(3 m, R)$ by $x \rho=\left(x_{i j}\right)$ where for $i, j=1,2, \cdots, m$ we have

$$
\begin{aligned}
x_{i j} & =a_{i} \tau & & \text { if } i \sigma=j, \\
& =0_{3 \times 3} & & \text { otherwise. }
\end{aligned}
$$

Then $\rho$ is a faithful representation of $G$. Clearly $x_{11}=0$ if $x \notin H$ and $x_{11}=x \tau$ if $x \in H$. Thus $g^{H} \rho$ is Zariski closed in $H \rho$ while $H \rho$ is Zariski open in $G \rho$. But $H$ has finite index in $G$, so $H \rho$, and hence $g^{H} \rho$, is Zariski closed in $G \rho$. Finally, $g^{G} \rho=\bigcup_{i} g^{H x_{i}} \rho$, so $g^{\theta} \rho$ is also Zariski closed in $G \rho$. 
COROLlaRY ([1], [5] and [6]). Free groups are conjugacy separable.

Proof. The ring $R=Z\left[X, Y^{-1}\right]$ of Theorem 1 is clearly an integral domain. Further, since it is isomorphic to the group ring of the free abelian (and hence residually finite) group on $Y$ over the polynomial (and hence residually finite) ring $Z[X \backslash Y]$ it follows that $R$ is a residually finite ring. The corollary now follows from Lemma 2 and Theorem 1.

One can squeeze a little more out of Theorem 1.

THEOREM 2. Let $G$ be a finite extension of a free group $H$ and $g$ an element of $G$ of infinite order. Then there exist a ring $R=Z\left[X, Y^{-1}\right]$, where $X$ is a set of indeterminates with $|X| \leqq|G|$ and $Y$ is a subset of $X$, and a faithful matrix representation $\rho$ of $G$ over $R$ of finite degree such that $g^{G} \rho$ is Zariski closed in $G \rho$.

Proof. Let $m=(G: H)$. By Theorem 1 there exist a ring $R$ of the required type, an integer $n$, and a faithful representation $\tau$ of $H$ into $G L(n, R)$ such that $\left(g^{m}\right)^{H} \tau$ is Zariski closed in $H \tau$. Just as in the final paragraph of the proof of Theorem 1 we may define a faithful representation $\rho$ of $G$ into $G L(m n, R)$ such that both $\left(g^{m}\right)^{G} \rho$ and $H \rho$ are Zariski closed in $G \rho$. Now the map $\mu: x \mapsto x^{m}$ of $G$ into itself is Zariski continuous. Hence $\left(g^{m}\right)^{G} \rho \mu^{-1}$ and $g H \rho$ are Zariski closed in $G \rho$ and consequently, by Lemma 3, so is $g^{G} \rho$.

Just as we obtained the corollary to Theorem 1 we can derive from Theorem 2 the following result of Stebe [6, Theorem 2].

COROLlary. If $G$ is a finite extension of a free group and $g$ is an element of $G$ of infinite order then $g$ is conjugacy distinguished in $G$.

Naturally one wonders for which conjugacy separable groups with faithful matrix representations is there an analogue of Theorem 1. Perhaps the most obvious groups to consider here are the polycyclic groups, and in particular the finitely generated nilpotent groups. The following is very easy to prove.

If $G$ is a finitely generated nilpotent group of class 2 , then for each element $g$ of $G$ there exists an integer $n$ and a faithful representation $\rho$ of $G$ into $G L(n, Z)$ such that $g^{G} \rho$ is Zariski closed in $G \rho$.

\section{REFERENCES}

1. G. Baumslag, Residual nilpotence and relations in free groups, J. Algebra 2 (1965), 271-282. MR 31 \#3487.

2. M. Hall, Coset representations in free groups, Trans. Amer. Math. Soc. 67 (1949), 421-432. MR 11, 322. 
3. W. Magnus, A. Karrass and D. Solitar, Combinatorial group theory. Presentations of groups in terms of generators and relations, Pure and Appl. Math., vol. 13, Interscience, New York, 1966. MR 34 \#7617.

4. V. L. Nisnevič, Uber Gruppen die durch Matrizen über einem kommutativen Feld isomorph darstellbar sind, Mat. Sb. 8 (50) (1940), 395-403. MR 2, 309.

5. V. N. Remeslennikov, Groups that are residually finite with respect to conjugacy, Sibirsk. Math. Ž. 12 (1971), 1085-1099=Siberian Math. J. 12 (1971), 783-792.

6. P. F. STEBe, A residual property of certain groups, Proc. Amer. Math. Soc. 26 (1970), 37-42. MR 41 \#5494.

7. B. A. F. Wehrfritz, Generalized free products of linear groups, Proc. London Math. Soc. (to appear).

8. - Infinite linear groups, Ergebnisse der Mathematik und ihrer Grenzgebiete, Band 76, Springer-Verlag, Berlin and New York, 1973.

Department of Mathematics, Queen Mary College, London, E1 4NS, England 